\title{
Herramienta Automatizada para documentos básicos de la contabilidad
}

\section{Automated Tool for Accounting 's basic documents}

\author{
Adriel Malvarez Cuello. ${ }^{1}$
}

\section{DOI: https://doi.org/10.33262/visionariodigital.v4i3.1272}

\begin{abstract}
.
The use of technological tools that provide support to the process of taking of decisions, it allows to the companies to obtain competitive advantages as consequence of the activation of the process that will rebound in a remarkable way in the general yield of the organization. The Automated Tool for Accounting's basic documents seeks to reach these results, without being left the traditional mark. Its creation, bornenfocad of the necessities of students, focused in negotiating in an efficient way its time and aspirations. Its complementation with the educational process is evident; benefitting, as much professors as students, with a bigger illustration and speed in the learning process. The reality of the link university-technology is clearer and more necessary everyday, betting hence meets the Tourism's Faculty of the University of Havana with the setting in practice of instruments of this type.
\end{abstract}

Keywords: Accounting, Automated Tool, time, learning.

\section{Resumen.}

El uso de herramientas tecnológicas que proporcionan soporte al proceso de toma de decisiones permite a las empresas obtener ventajas competitivas como consecuencia de la agilización del proceso que repercutirá de manera notable en el rendimiento general de la organización. La Herramienta Automatizada para documentos básicos de Contabilidad y Finanzas pretende alcanzar estos resultados, sin salirse del marco tradicional. Su creación, parte de las necesidades de estudiantes, centrados en gestionar de forma eficiente su tiempo y aspiraciones. Su complementación con el proceso docente es evidente; beneficiándose, tanto profesores como estudiantes, con una mayor ilustración y rapidez en el proceso de aprendizaje. La realidad del vínculo universidad-tecnología es

\footnotetext{
1 Facultad de Turismo-Universidad de La Habana, Cuba, email adriel_m@estudiantes.ftur.uh.cu, adrielmalva2017@gmail.com
} 
cada día más clara y necesaria, apostando por ello se encuentra la Facultad de Turismo de la Universidad de La Habana con la puesta en práctica de instrumentos de este tipo.

Palabras clave: Contabilidad, Herramienta Automatizada, tiempo, aprendizaje.

\section{Introducción.}

Las empresas en los actuales momentos están en permanente evolución tecnológica, necesitan de un sistema de investigación instantáneo que lleve a cualquier miembro que solicite la referencia económica a obtenerla de una manera rápida, de este modo se dispone de gran cantidad de información, con lo cual los negocios están en condiciones de realizar notables progresos en materia de eficiencia, crecimiento y beneficios. En muchas empresas la información se está convirtiendo en el factor más importante que diferencia a las compañías exitosas de las que no lo son.

En la rama de la contabilidad es notable el avance que ha provocado en las empresas, el procesamiento de los datos primarios por medio del uso de sistemas informáticos, se ha beneficiado mucho de la tecnología de la computación incluso más que cualquier otra área de negocio. La aplicación de la computadora en la contabilidad es algo natural porque estos equipos son ideales para realizar cálculos repetitivos.

Los softwares contables se han convertido en una herramienta de información económica necesaria dentro de los negocios. En una organización se puede ver la información de distintas maneras, según el interés de su departamento. Así, el gerente pedirá los reportes financieros y de ventas para conocer la realidad de la organización y tomar decisiones oportunas.

Los sistemas computarizados permiten al personal de una empresa optimizar el tiempo para emplearlo en otras actividades a favor del negocio, también dar valor agregado a otros servicios debido a la agilidad de la información.

La información solicitada en la parte contable requiere de un sistema que sea diseñado para servir de una manera fácil y rápida, pero a la vez que proporcione la información oportuna y detallada sobre la situación financiera de un negocio

Los sistemas informáticos empiezan a revolucionar la propia naturaleza de la actividad económica, permiten entregar reportes de ventas y existencias para cada uno de los productos o servicios y entregan estadísticas inmediatas de ventas y existencias en cualquier parte del mundo.

A pesar del desarrollo del tema a nivel mundial. En el país existen pocos y en la Facultad de Turismo de la Universidad de La Habana, no existe un software contable. De ahí que se plantea como problema el cómo agilizar el aprendizaje del proceso contable en la Facultad de Turismo de La Universidad de La Habana. 
Se conoce como Tecnología de información y comunicación a la utilización de técnicas en computadoras y ordenadores electrónicos para el manejo y procesamiento de información, específicamente la captura, transformación, almacenamiento, protección, y recuperación de datos e investigación.

Los orígenes de la TIC son recientes; aunque el nombre de Tecnología de la Información se inicia en la década de 1970. Su utilización en los negocios se remonta a mediados del siglo XX, durante la segunda guerra mundial; sin embargo, es en los últimos 20 años donde alcanza niveles de uso y aplicaciones tan variadas, que se ha convertido en un área de gran amplitud e impacto en todos los aspectos de la vida cotidiana, incluyendo la gerencia de cualquier empresa, en la cual hoy en día es casi indispensable.

La evolución de las TIC y el desarrollo de la indagación contable han seguido caminos paralelos; la contabilidad, como sistema de investigación empresarial registra la información de las actividades que realizan las empresas y, mediante los estados bancarios, ofrece un reflejo de la imagen fiel de su patrimonio, su situación financiera y sus resultados. (Weickgenannt \& Turner, 2008) La aplicación de las TIC en este ámbito, por tanto, tiene diferentes efectos: por un lado, el avance en los instrumentos y programas informáticos supone un registro más rápido y más actualizado de las operaciones de la empresa.

La indagación registrada está disponible y puede ser utilizada en cualquier momento para decisiones de la entidad. Por otro lado, la transferencia de investigación desde el exterior procedente de proveedores, clientes, administraciones públicas y otros organismos, como los reguladores de la información contable, es inmediata, lo que permite que pueda estar permanentemente actualizada.

La importancia del desarrollo de software se basa en su capacidad de optimizar la productividad de otros sectores a través de la tecnología y mejoramiento de procesos a base de la industria y una economía basada en servicios. El desarrollo del sector, software y la industria de las TIC están llevando a los países menos desarrollados, a un camino de adelanto, modernización, conectividad a nivel global y crecimiento económico.

Más allá, la producción de software y la prestación de servicios, son actividades económicas cada vez más importantes, capaces de crear empleos calificados y generar divisas por intermedio de las exportaciones de productos y servicios a distancia; esta oportunidad se hizo posible a partir de los avances tecnológicos en las áreas de comunicación y arquitectura de sistemas ocurridos en la última década.

\section{Desarrollo.}

En el mercado mundial hay una amplia variedad de paquetes de programas enfocados a la gestión administrativa contable. Las marcas se esmeran por entregar múltiples ventajas 
y beneficios a los empresarios. Sin embargo, algunos se han venido ubicando mejor y definiendo con precisión, sus públicos objetivos.

Una de sus grandes diferenciadores es el precio, otro, si es un software por suscripción online o de licencia vitalicia y, en resumen, sea cual sea el esquema, todos están comprometidos en brindar un orden a la administración de la empresa. Cuba, debido a restricciones de pago por internet y limitaciones que impone el criminal bloqueo económico, presenta dificultades a la hora de adquirir o utilizar estos programas. Sin embargo, existen softwares libres o gratuitos, pero en su mayoría son versiones de prueba o herramientas limitadas.

La prensa nacional confirma que, Cuba dispone de 38 sistemas contables informatizados certificados, de ellos, ocho son de producción nacional, pero aún la contabilidad no se encuentra totalmente informatizada en el país.

Una herramienta digital para llevar por cuenta propia la contabilidad de quienes poseen una licencia operativa en el sector no estatal fue desarrollada y puesta a la venta por la Empresa de Tecnologías de la Información y Servicios Telemáticos Avanzados (Citmatel), bajo el nombre Administre su negocio.

Se evidencia el empeño del país por avanzar en este sentido pese a las dificultades, observándose también la iniciativa en el sector no estatal. La pequeña empresa por sus características de menor rango de operaciones y mayor centralización de recursos, presenta facilidades de aplicación para este tipo de instrumentos. Considerándose también que podrían ajustarse de una manera más específica a los requerimientos necesarios para su gestión.

En el caso de la universidad, se conoce que no es notable la presencia de los softwares contables. Aunque los estudiantes puedan adquirir o conocer de alguno por sus propios medios esto no garantiza que se adecue al contenido que necesita, ya que generalmente los softwares contables muestran salidas ya elaboradas y no documentos detallados como el procedimiento manual tradicional. Por lo tanto, este es un limitante que sin lugar a dudas obstaculiza el vínculo universidad-tecnología.

La situación actual que presenta la Facultad de Turismo de la Universidad de La Habana es que carece de un software contable. Sin embargo, el colectivo de profesores de la asignatura Contabilidad y Finanzas afirman que es una necesidad inminente dado el contexto actual y la relación universidad-tecnología. Además, podría y debería ser incluido en las temáticas de la asignatura con el objetivo de facilitar la impartición de contenidos, propiciando a su vez un mejor y más rápido aprendizaje. 


\section{Metodología.}

La herramienta se diseñada mediante el programa Microsoft Excel y utiliza las características fundamentales de las hojas de cálculo para lograr la automatización del proceso contable en cuestión. Las combinaciones de fórmulas utilizadas y las referencias entre diferentes hojas de cálculo brindan mayor facilidad y rapidez, sin la necesidad de recursos informáticos de avanzado nivel tecnológico.

La fundamentación teórica de la herramienta propuesta se encuentra en ciencias como Contabilidad y Finanzas, destacando la confección de documentos básicos de contabilidad, la información brindada por las razones financieras, ecuaciones necesarias, entre otras.

\section{Resultados.}

La herramienta se constituye a través de hojas de cálculo, en un orden lógico o algoritmo de trabajo, siendo este el utilizado a la hora de realizar el proceso contable de forma manual. Haciendo que los datos introducidos en la primera hoja sean necesarios para trabajar en la segunda, y así sucesivamente hasta el final, que sería la situación financiera de la empresa según sus ratios financieros.

Las ventajas que aporta la implementación de este sistema según el autor de esta investigación son:

- A diferencia de un software de contabilidad como el Express Accounts u otros, esta herramienta brinda la posibilidad de realizar el proceso contable de la manera tradicional sin perder el algoritmo de trabajo que deben realizar los estudiantes en las clases. Esto genera gran ahorro de tiempo en las clases prácticas y la posibilidad de aclarar mayor cantidad de dudas.

- La herramienta fue diseñada desde la perspectiva del estudiante, facilitando esto el entendimiento por parte de otros en igual condición.

- No es necesario realizar operaciones matemáticas, o muy pocas en caso de hacer alguna comprobación. Generando así mayor velocidad en la realización de los documentos.

- La herramienta es capaz de determinar en la mayoría de las ocasiones, el lugar donde se introdujo mal un dato, o el caso en que falla algo y, por lo tanto, la rectificación de errores (aunque debe realizarse de forma manual) es sencilla, antecedida por la previa detección del error.

- No se necesitan conocimientos avanzados en informática o para la utilización de Microsoft Excel, con un conocimiento básico es suficiente. Además, cuenta con 
tutoriales explicativos que demuestran cómo utilizar la herramienta y el procedimiento de introducción de datos en Excel.

- Cuenta además con información útil en varios documentos, lo que facilita determinar estados de las empresas o analizar ratios financieras, sin necesidad de acudir a la consulta de bibliografía independiente.

Las limitaciones que aporta la implementación de este sistema según el autor de esta investigación son:

- Incapacidad de autocorrección. Pese a la detección del error, la corrección debe ser manual

- Necesaria introducción de datos en el lugar específico y no existencia de un área donde ubicar las cuentas de forma aleatoria y que la herramienta las organice y procese.

Para una comprensión plena de este trabajo se divide por hojas la explicación de cada documento básico que forma parte de este procedimiento.

Libro Diario: En la mayoría de los casos el Libro Diario suele tener un gran número de asientos o registros. La sumatoria de estos se hace por hoja y al pasar a otra hoja ya las sumas de débitos y créditos de la anterior están realizadas y presentes en la nueva hoja. Sin embargo, un error en algún asiento a veces es difícil de detectar y requiere una búsqueda minuciosa, o de la repetición de todo el proceso contable lo que resulta muy engorroso sobre todo para aquellos estudiantes o contadores que no cuentan con una vasta experiencia.

Esta situación genera una gran pérdida de tiempo, agregándole lo trabajoso que resulta sumar todos los importes de las cuentas que se registran. La herramienta automatizada se limita a solo la introducción de las cuentas y sus importes, sumando automáticamente los mismos. Mientras que, en caso de algún error, esta detecta el asiento en que este se encuentra. Tal como se muestra en la figura 1. 
Figura 1. Hoja de Cálculo de Libro Diario
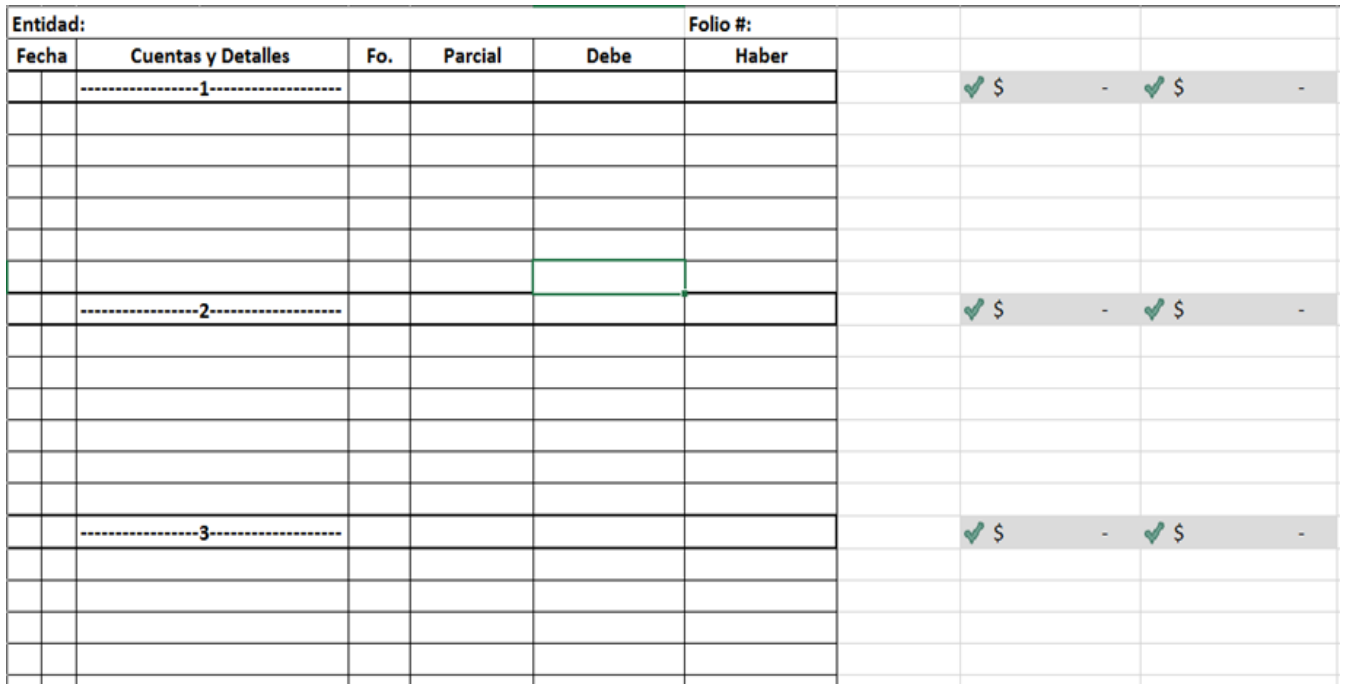

Fuente: Elaboración propia.

Libro Mayor: Las operaciones se registran por primera vez en el Libro Diario, luego de haber cumplido este paso debemos realizar lo que se denomina pases al Mayor; esto consiste que todos los asientos de diario deben pasarse a medida que se van sucediendo al mayor, cuenta por cuenta, o sea el proceso de transferir y distribuir al mayor es lo que se denomina pase, proceso mediante el cual se obtiene el saldo de las cuentas. (Maldonado)

Este procedimiento se hace utilizando la Cuenta T, pero esto es generalmente académico, para ahorrar tiempo y por su facilidad.

La herramienta solo requiere de la introducción del débito o crédito tal y como se registró en el Libro Diario, en cada una de las cuentas que representa, y esta determina el saldo. Para ello es necesario conocer previamente la naturaleza de las cuentas.

Figura 2. Modelo de Cuenta $\mathrm{T}$ en hoja de Cálculo. Una para cada cuenta registrada.

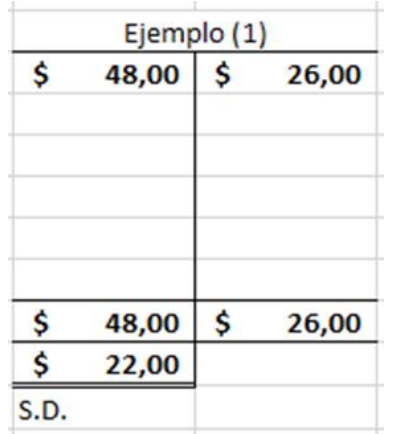

Fuente: Elaboración propia. 
Balance de comprobación: Una vez determinados los saldos de las cuentas en el Mayor General, el siguiente paso será la elaboración del Balance de Comprobación.

Bien sabemos que toda operación conlleva un intercambio de valores en sentido contrario; toda transacción origina por lo menos un cargo y un abono por importes iguales; si esto se cumple en el registro de cada operación, el conjunto de todas las operaciones debe dar como resultado un equilibrio entre todos los cargos y todos los abonos. (Maldonado)

Así entonces se puede concebir como el documento comprobatorio de todo el registro realizado en los libros básicos, mostrando todos los saldos y totales de las cuentas que intervienen. Con solo la introducción de las cuentas y sus importes, en esta hoja de la herramienta quedará conformado este documento. Además, el documento mostrará con una (x), la casilla final si los débitos son diferentes de los créditos.

Figura 3. Hoja de Cálculo de Balance de Comprobación.

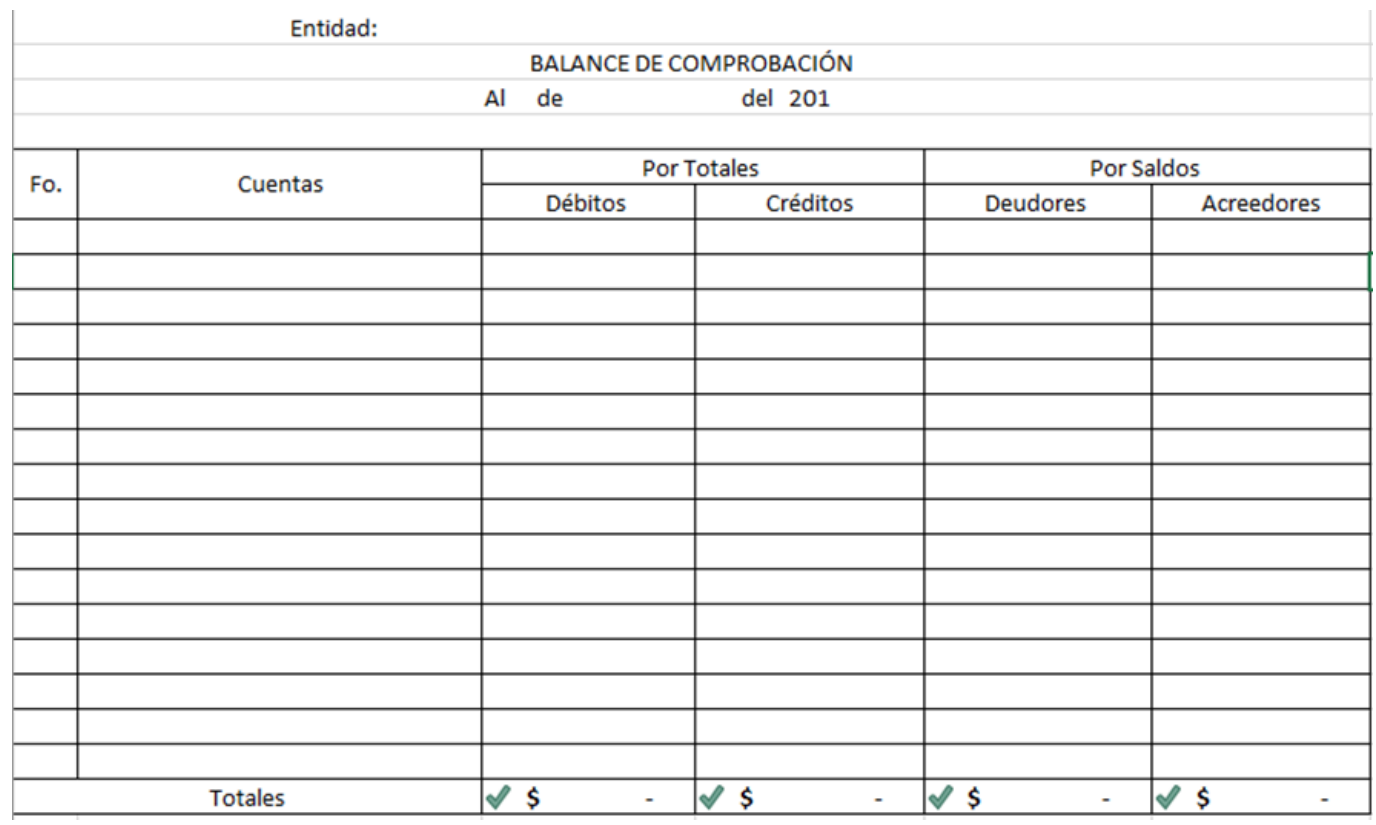

Fuente: Elaboración propia.

Asientos de Cierre: Los Asientos de Cierre no son documentos diferenciados, son la parte final del Libro Diario en un período de tiempo determinado. Pero surge la necesidad de dedicarle un apartado dadas sus características.

En primer lugar, como su nombre lo indica va a contabilizar el cierre del período. Se dejarán saldadas todas las cuentas acreedoras y deudoras. Finalmente es aquí donde se acuña el capital de la empresa al final del ejercicio. El procedimiento de confección en la herramienta es similar al del libro diario, pero es necesario hacer algunas concesiones y cálculos adicionales. Esto está implícito y descrito en la propia hoja de cálculo correspondiente. Cabe destacar que un ejercicio pude tener tres finales posibles: ganancia, 
pérdida o ninguno. Para cada caso existe un método de resolución diferente donde influye además la existencia o no, de una cuenta personal a favor del propietario.

\section{Balance General y Estado de Resultados:}

El Balance General, junto al Estado de Resultados, constituye el informe contable más importante que debe elaborar la empresa al finalizar cada ejercicio contable; en este caso se trata de presentar en forma clasificada todas las cuentas que representan bienes, derechos, obligaciones y el capital para una fecha dada, es decir una relación de las cuentas reales, de orden y de valuación que presenten la situación de la empresa en marcha para la fecha de cierre.

El Estado de Resultados, a diferencia, recoge las cuentas nominales (Ingresos y Gastos) dado su carácter perecedero y efímero, lo que claramente las diferencias de las cuentas reales. Define la utilidad neta del ejercicio, después de analizar todas las cuentas referentes.

Ambos documentos se realizan de forma similar, siendo necesario un estudio previo de realización de los mismos. Es necesaria la introducción de cuentas y la colocación de valores en las filas correspondientes.

Figura 4. Hoja de Cálculo de Balance General.

\begin{tabular}{|c|c|c|c|c|c|c|c|}
\hline \multicolumn{8}{|c|}{ Empresa X } \\
\hline \multicolumn{8}{|c|}{ Balance General } \\
\hline \multicolumn{3}{|c|}{ Al $\perp \_$} & & & & & \\
\hline Activos & & & Pasivos: & & & & \\
\hline Circulantes: & & $\$$ & Circulantes: & & & $\$$ & . \\
\hline Efectivo en Caja & & & Efectos por Pagar a Corto Plazo & & $\$$ & & \\
\hline Efectivo en Banco & & & Cuentas por Pagar Proveedores & & & & \\
\hline Efectos por Cobrar a Corto Plazo & & & Cuentas por Pagar Diversas & & & & \\
\hline Cuentas por Cobrar Clientes & & & Intereses por pagar & & & & \\
\hline Pagos Anticipados & & & Impuestos por Pagar & & & & \\
\hline Mercancías & & & A largo Plazo: & & & & 0,00 \\
\hline Fijo Tangible & & 0,00 & Efectos por Pagar a Largo Plazo & & & & \\
\hline Terreno & & & Hipoteca por Pagar & & & & \\
\hline Edificio & & & Créditos Diferidos & & & & 0,00 \\
\hline Menos:Depreciación Acum. del Edificio & 0,00 & & Alquileres cobrados por anticipado & & & & \\
\hline Vehículo & & & Intereses cobrados por anticipado & & & & \\
\hline Menos:Depreciación Acum. del & 0,00 & & Total de Pasivos & & & $\$$ & - \\
\hline Mobiliario & & & Capital & & & & \\
\hline Menos : Depreciación Acum. Del & 0,00 & & X,Capital & 0 & & & \\
\hline Fijo Intangible & & 0,00 & Más:Utilidad Neta del ejercicio & $\underline{0}$ & 0 & & \\
\hline Plusvalia & & & Menos:Cuenta Personal & & 0 & & \\
\hline Marca de Fábrica & & & Nuevo Monto del Capital & & & $\$$ & \\
\hline Patente de Inversión & & & Total de pasivos y capital & & & $\$$ & - \\
\hline $\begin{array}{l}\text { Menos: Amortización Acum. Patente de } \\
\text { Inversión }\end{array}$ & 0,00 & & & & & & \\
\hline Gastos Diferidos & & $\$$ & & & & & \\
\hline \multicolumn{8}{|l|}{ Campaña Publicitaria } \\
\hline Gastos de Organización & & & & & & & \\
\hline Total de Activos & & $\$$ & & & & & \\
\hline
\end{tabular}

Fuente: Elaboración propia. 
Figura 5. Hoja de Cálculo de Estado de Resultados.

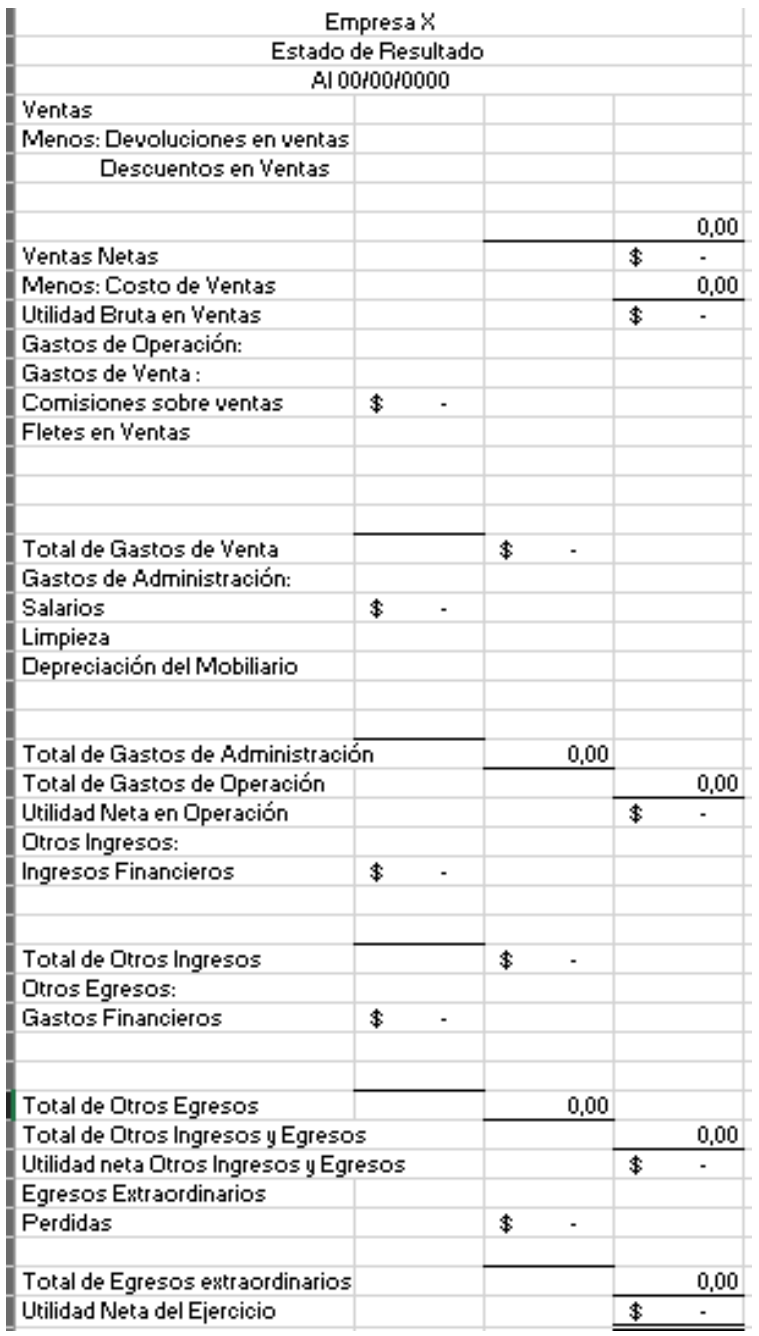

Fuente: Elaboración propia.

\section{Análisis Vertical y Horizontal de los Estados Financieros:}

El Análisis Vertical, es la evaluación del funcionamiento de la empresa en un periodo ya especificado. Este análisis según sirve para poner en evidencia la estructura interna de la empresa, también permite la evaluación interna y se valora la situación de la empresa con su industria. (Universidad Interamericana Para El Desarrollo)

El Análisis Horizontal se realiza con Estados Financieros de diferentes periodos, quiere decir de diferentes años y se examina la tendencia que tienen las cuentas en el transcurso del tiempo ya establecido para su análisis. El cálculo de este análisis se realiza con la diferencia de dos o más años para tener como resultado valores absolutos y con porcentaje tomando un año base para poder tener datos relevantes que analizar. En dado caso que se haga el examen con los valores absolutos o los porcentajes, podría resultar un error en la toma de decisiones. 
Con los datos pertinentes introducidos, solo se necesita interpretar los resultados de cada tipo de análisis.

Figura 6. Hoja de Cálculo para Análisis vertical y Horizontal de los estados de resultado de dos períodos. Semejante al mismo análisis del Balance General.

\begin{tabular}{|c|c|c|c|c|c|c|}
\hline \multirow{2}{*}{ Indicadores } & \multirow{2}{*}{ Año Actual } & & \multirow{2}{*}{ Año Anterior } & & \multirow[b]{2}{*}{ Variación Absoluta } & \multirow[b]{2}{*}{ Variación Relativa } \\
\hline & & & & & & \\
\hline Ventas Netas & & $\#$ \#DIV/o! & & $\#$ \#DIV/o! & 0 & $\#$ \#DIV/O! \\
\hline$(-)$ Costo de Ventas & & $\#$ \#IDIV/0! & & \#iDIV/0! & 0 & \#iDIV/O! \\
\hline Utilidad Bruta en Operaciones & 0 & $\# i D I V / 0 !$ & 0 & $\#$ \#DIV/O! & 0 & $\#$ \#DIV/O! \\
\hline (-) Gastos de operaciones & & $\#$ \#IV/0! & & $\#$ \#IV/0! & 0 & $\#$ \#DIV/O! \\
\hline Utilidad Neta en Operaciones & 0 & $\#$ \#IVIV/0! & 0 & $\#$ \#IVIV/0! & 0 & $\#$ \#DIV/O! \\
\hline \multicolumn{7}{|l|}{ ( \pm Resultados Extraordinarios } \\
\hline Utilidades antes intereses e impuestos & 0 & $\#$ \#DIV/O! & 0 & \#iDIV/o! & 0 & \#DIV/O! \\
\hline (-) Intereses & & $\#$ \#DIV/0! & & $\#$ \#DIV/0! & 0 & $\#$ \#DIV/O! \\
\hline Utilidad antes impuestos & 0 & $\#$ \#IDIV/0! & 0 & $\#$ \#IDIV/0! & 0 & $\#$ \#IVIV/O! \\
\hline (-) Impuestos & & $\#$ \#IV/O! & & $\#$ \#IV/O! & 0 & $\#$ \#IV/O! \\
\hline Utilidad Neta & 0 & $\#$ \#DIV/o! & 0 & $\#$ \#DIV/o! & 0 & $\#$ \#DIV/O! \\
\hline (-)Dividendos & & $\#$ \#IVIV/0! & & $\#$ \#DIV/0! & 0 & \#iDIV/o! \\
\hline Unidades Retenidas & 0 & $\#$ \#IDIV/0! & 0 & \#iDIV/0! & 0 & \#iDIV/O! \\
\hline
\end{tabular}

Fuente: Elaboración propia.

Razones Financieras: Una razón es una simple expresión matemática de la relación de una partida con otra; es el cociente entre magnitudes que tienen una cierta relación y por este motivo se comparan.

Para calcular una razón significativa debe existir una relación significativa entre las dos cifras, de manera tal que, para variar el comportamiento del resultado de la razón, se pueda tomar una acción correctiva sobre cualquiera de las partidas que la determinaron, o sobre ambas, aunque una interpretación completa de una razón frecuentemente requiere investigación adicional de los datos básicos. Las razones representaron una ayuda para el análisis e interpretación, pero no sustituyen un buen juicio analítico.

Al analizar cambios en pesos, en porcentajes y en razones, los analistas financieros constantemente buscan algún patrón de comparación (estándares de comparación) que pueda utilizarse para juzgar si las relaciones que han encontrado son favorables o no.

No es necesaria la introducción de datos, una vez realizados los análisis verticales y horizontales, se podrán determinar las razones financieras. No es necesario conocer los valores ideales de cada razón, basta con colocar el cursor encima del resultado y se mostrarán los valores requeridos. Además de que las celdas verdes contendrán los resultados favorables, las rojas los valores negativos o peligrosos y las amarillas los relativamente neutrales o de exceso. 
Figura 7. Hoja de Cálculo de Razones Financieras. Ejemplo aleatorio de empresa con mala actividad comercial.

\begin{tabular}{|c|c|c|}
\hline Ratios Liquidez & Año Actual & Año Anterior \\
\hline General & 2,42 & 3,85 \\
\hline Inmediata $\quad A C-$ Existencias/ $P C$ & 2,22 & 3,56 \\
\hline Efectivo/PC & 2,15 & 3,46 \\
\hline \multicolumn{3}{|c|}{ Ratios de Solvencia } \\
\hline Ratio de Solvencia AT/ Deuda & 1,64 & 2,04 \\
\hline \multicolumn{3}{|c|}{ Ratios de Endeudamientos } \\
\hline $\begin{array}{l}\text { Razón de Endeudamiento Deudas } \\
\text { totales/AT }\end{array}$ & 0,61 & 0,49 \\
\hline $\begin{array}{l}\text { Razón de Autonomia } \quad \text { Recursos } \\
\text { Propios/ AT }\end{array}$ & 167,66 & 2,48 \\
\hline $\begin{array}{l}\text { Razón de Pasivo Total a Capital } \\
P T / C+P T\end{array}$ & 0,00 & 0,16 \\
\hline Calidad de Deudas PC/PT & 0,63 & 0,50 \\
\hline \multicolumn{3}{|l|}{ Ratios de Actividad } \\
\hline $\begin{array}{l}\text { Rotación de inventarios } \\
\text { Ventas/Inventarios }\end{array}$ & 402,30 & 491,67 \\
\hline Duración del ciclo de Inventarios & 0,89 & 0,73 \\
\hline $\begin{array}{l}\text { Rotación de Cuentas por Cobrar Ventas/ } \\
\text { Clientes }\end{array}$ & 1196,58 & 1573,33 \\
\hline Duración del ciclo de cobro & 0,30 & 0,23 \\
\hline Rotación de Activos Fijos Ventas/AF & 466,67 & 662,18 \\
\hline $\begin{array}{l}\text { Rotación de Activos Total } \\
\text { Ventas/AT }\end{array}$ & 31,01 & 35,91 \\
\hline
\end{tabular}

Fuente: Elaboración propia.

\section{Conclusiones:}

La presente investigación permitió arribar a las siguientes conclusiones:

- El uso de herramientas tecnológicas que proporcionan soporte al proceso de toma de decisiones permite a las empresas obtener ventajas competitivas como consecuencia de la agilización del proceso que repercutirá de manera notable en el rendimiento general de la organización.

- Procesos que datan de siglos anteriores, pueden ser llevados al mundo digital, sin perder su algoritmo de ejecución. Resultado coherente y relativo al incuestionable desarrollo de la tecnología.

- La Herramienta Automatizada para documentos básicos de Contabilidad y Finanzas surge desde y para los propios estudiantes de la Facultad de Turismo de 
la Universidad de La Habana. Como un sistema enfocado en apoyar y mejorar las clases de Contabilidad y Finanzas

\section{Referencias bibliográficas:}

Acosta, D. A., \& Navarrete, G. (2013). Importancia del uso del software contable en pequeñas, medianas y grandes empresas del cantón Portoviejo. La Técnica (10), 62-72.

Bocanera, R. (2005). Historia de un sistema contable que revolucionó el mundo. Petrotecnia, 98-103.

Brighan, E. F., \& Houston, J. F. (2006). Fundamentos de Administración Financiera. México: CECSA.

Crean software para administrar contabilidad de negocios de trabajadores no estatales cubanos. (2016). Juventud Rebelde. Obtenido de http://www.cadenagramonte.cu/: http://www.cadenagramonte.cu/

Daivid, R. (2008). Conceptos de Administración Estratégica. México: Pearson Prentice Hall.

Fernández, R. (s.f.). COSTOS Y GASTOS De lo elemental a lo fundamental. La Habana: Escuela de Altos Estudios de Hotelería y Turismo.

Gates, B. (1999). Los negocios en la era digital. España: Plaza\&Janés.

Maldonado, R. (s.f.). Estudio de la Contabilidad General.

Padilla, G. (s.f.). Introducción al estudio de la información financiera. México: UNAM FCA Publishing.

Romero López, Á. J. (s.f.). Principios de Contabilidad (5ta ed.). Ed.McGraw Hill.

Shim, J. K., \& Siegel, J. G. (2004). Dirección Financiera. España: Mc Graw Hill.

UNIVERSIDAD INTERAMERICANA PARA EL DESARROLLO. (s.f.). Análisis Financiero.

Uribe, J. (11 de enero de 2017). Software Contable: El Impacto de la tecnología en la contabilidad.... ¿Desaparecerá la contabilidad tal y como la conocemos? ¡Lectura especialmente apta para contadores! Obtenido de Blog-Loggro: https://www.loggro.com/

Vilet, G. (2004). La Administración Basada en la Tecnología de la información. Obtenido de http://edipo.madm.uaslp.mx/

Weickgenannt, A., \& Turner, L. (2008). Accounting Information Systems: Controls and Processes. John Wiley \& Sons.

Weston, J. F. (1992). Fundamentos de Administración Financiera (Vol. II.). Los Ángeles, California. 


\section{PARA CITAR EL ARTÍCULO INDEXADO.}

Malvarez Cuello, A. (2020). Herramienta Automatizada para documentos básicos de la contabilidad. Visionario Digital, 4(3), 48-61.

https://doi.org/10.33262/visionariodigital.v4i3.1272

\section{\Ciencia}

El artículo que se publica es de exclusiva responsabilidad de los autores y no necesariamente reflejan el pensamiento de la Revista Visionario Digital.

El artículo queda en propiedad de la revista y, por tanto, su publicación parcial y/o total en otro medio tiene que ser autorizado por el director de la Revista Visionario Digital.
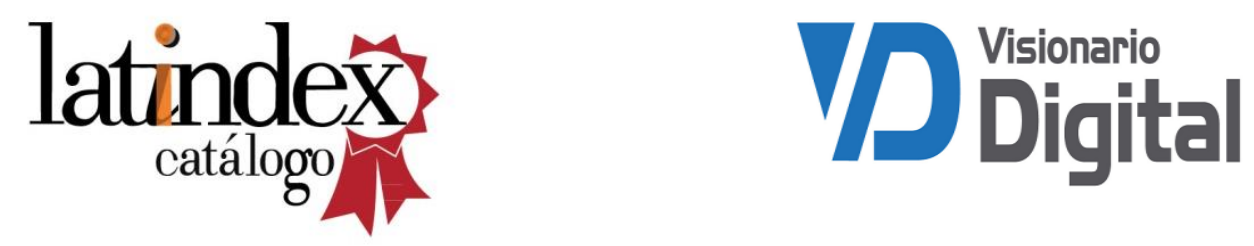